\title{
A Bayesian Approach to the Determination of Ignition Delay
}

\author{
Timothy Bodisco*, Samantha Low Choy, Richard J. Brown \\ 2 George St, Science and Engineering Faculty, Queensland University of Technology, \\ Brisbane, Australia, 4000
}

\begin{abstract}
A novel in-cylinder pressure method for determining ignition delay has been proposed and demonstrated. This method proposes a new Bayesian statistical model to resolve the start of combustion, defined as being the point at which the band-pass in-cylinder pressure deviates from background noise and the combustion resonance begins. Further, it is demonstrated that this method is still accurate in situations where there is noise present. The start of combustion can be resolved for each cycle without the need for ad hoc methods such as cycle averaging. Therefore, this method allows for analysis of consecutive cycles and inter-cycle variability studies. Ignition delay obtained by this method and by the net rate of heat release have been shown to give good agreement. However, the use of combustion resonance to determine the start of combustion is preferable over the net rate of heat release method because it does not rely on knowledge of heat losses and will still function accurately in the presence of noise. Results for a six-cylinder turbo-charged common-rail diesel engine run with neat diesel fuel at full, three quarters and half load have been presented. Under these conditions the ignition delay was shown to increase as the load was decreased with a significant increase in ignition delay at half load, when compared with three quarter and full loads.
\end{abstract}

Keywords: Ignition delay, diesel engine, Bayesian modelling, Markov-chain Monte Carlo, net rate of heat release

\footnotetext{
*Corresponding Author.

e-mail: timothy.bodisco@qut.edu.au
} 


\section{Introduction}

Standard methods for determining the start of combustion have changed little in the last few decades. Most current studies [1-7] that examine the start of combustion, or ignition delay, use the net rate of heat release, with most citing the 1988 book Internal Combustion Engine Fundamentals written by John Heywood [8]. This method is commonly used because it is considered reliable and the net rate of heat release is simple to calculate. However, this paper will introduce the use of a statistical model in the Bayesian paradigm to accurately determine the start of combustion.

Calculation of the net rate of heat release comes from analysing the heat losses in an engine from a first law of thermodynamics perspective, in its most commonly used form [8]:

$$
\frac{d Q_{n}}{d t}=\frac{\gamma}{\gamma-1} p \frac{d V}{d t}+\frac{1}{\gamma-1} V \frac{d p}{d t}
$$

where, $\frac{d Q_{n}}{d t}$ is the net rate of heat release, $\gamma$ is the ratio of specific heats, $p$ is the in-cylinder pressure, $V$ is the in-cylinder volume and $t$ is time. More complicated versions of Equation 1 exist that take into account heat loss to the walls, effects of crevice regions and other possible sources for heat losswhich are mostly engine specific and not general. The start of combustion is defined as the point when the net rate of heat release begins increasing rapidly - some authors use the point that the net rate of heat release becomes positive [6].

From experimental in-cylinder pressure, another method for determining the start of combustion is from the rate of pressure rise [9]. This method locates the point at which the rate of pressure rise begins to increase rapidly, and can be done by analysing either the first or second derivative of the incylinder pressure signal. It has parallels with the net rate of heat release, which also requires the differentiation of the in-cylinder pressure data.

In a recent study by Rothamer and Murphy [10], six methods of determining the ignition delay were compared. The six methods used were:

1. location of $50 \%$ of pressure rise due to premixed burn combustion;

2. extrapoliation of the peak slope of pressure rise due to combustion to the zero crossing point;

3. location of the first peak of the second derivative of the pressure trace;

4. location of the first peak of the third derivative of the pressure trace; 
5. location of $10 \%$ of the maximum heat release rate in the premixed burn; and,

6. a repeat of (5) using a low-pass (threshold $2000 \mathrm{~Hz}$ ) filtered in-cylinder pressure trace.

Their study focused on jet fuels and diesel fuel in a heavy-duty directinjection single-cylinder diesel and the data analysis was performed using 250 cycles of averaged data. A conclusion from their study found that the methods which required second or third derivatives were not optimal owing to the presence of noise and that the ignition delay determined by the heat release method using the low-pass filtered in-cylinder pressure signal gave a result 200-330 $\mu$ s shorter than the other methods.

An early method for estimating ignition delay was proposed by Hardenberg and Hase [11]. They developed an empirical relationship between the parameters which they determined had the most impact on ignition delay: mean piston speed $(\mathrm{m} / \mathrm{s}), M_{P S}$; in-cylinder temperature at the time of injection $(\mathrm{K}), T$; the compression ratio, $r_{c}$; the polytropic index of compression, $n$; the cetane number, $C N$; and, the absolute charge-air pressure at the time of injection (bar), $P$. In crank-angle degrees the empirical relationship as determined by Hardenberg and Hase is:

$$
I D=\left(0.36+0.22 M_{P S}\right) e^{\frac{618840}{C N+25}\left(\frac{1}{R T}-\frac{1}{17190}\right)+\left(\frac{21.2}{P-12.4}\right)^{0.063}}
$$

where, $R$ is the universal gas constant $(8.31434 \mathrm{~J} /$ mole). The polytropic index of compression, $n$, and the compression ratio, $r_{c}$, impact on the temperature and pressure of the charge-air. Estimates of $T$ and $P$ can be obtained from the inlet manifold conditions $[8,11]$.

$$
\begin{aligned}
T & =T_{i} r_{c}^{n-1} \\
P & =P_{i} r_{c}^{n}
\end{aligned}
$$

Later work done by Prakash et al. [12] extended this model to incorporate dual-fuel operation of diesel engines.

Since the work done by Hardenberg and Hase, other estimators of ignition delay based on engine parameters have been developed. Assanis et al. [13] has extensively reviewed these and proposed their own method for estimating ignition delay. However, for experimentally validating their ignition delay estimator, Assanis et al. compared their estimator to measured values by taking the peak of the second derivative to be the start of combustion. 
Flame luminosity is another method used by researchers for determining the start of combustion. Heywood [8] argues that the use of flame luminosity detectors as a means to determine the start of combustion increases the potential for error. This is because the first appearance of the flame occurs after the increase in pressure. However, a recent study has argued that the first appearance of the flame coincides well with results from analysing the net rate of heat release [14]. Perhaps, with improving technology this method is becoming more reliable. Flame luminosity sensors are, however, prohibitively expensive for wide-spread practical use.

All of these approaches have practical difficulties or offer little, or no, information regarding cycle-by-cycle changes and hence do not allow for intercycle variability studies. In this paper a methodology for determining the start of combustion is proposed that requires no knowledge of difficult to estimate parameters such as heat loss to the walls and is still accurate with noisy data. Using only the in-cylinder pressure signal a statistical modelling approach is used to determine the start of combustion. A Bayesian approach to statistical modelling is used because it estimates the plausible range of parameter values (which includes the start of combustion), given the data observed [15]. In contrast a classical statistical analysis would provide the logical reverse: being estimates of the plausibility of the data, under specific (null or alternative) hypothesis of the parameter values. The latter would be better suited to confirmatory analyses where experimentalists wished to confirm whether parameter values took on specific values in a new situation. In this paper a new Bayesian modelling framework which provides posterior estimates of the start of combustion is given and is implemented across 4000 consecutive cycles at various engine loads to demonstrate its utility.

\section{Experimental Configuration}

Experiments were conducted at the QUT Biofuel Engine Research Facility (BERF) in June 2011. Table 1 contains the technical specifications of the engine and data acquisition equipment. The engine was run at $2000 \mathrm{rpm}$ on neat automotive diesel at full load $(760 \mathrm{Nm})$ and at three quarters $(570 \mathrm{Nm})$ and half $(380 \mathrm{Nm})$ of full load.

\section{Experimental Data}

Band-pass filtering was applied directly to the in-cylinder pressure signal, Figure 1 is an example of the in-cylinder pressure signal, prior to digitising. 
Table 1: Engine and data acquisition specifications

\begin{tabular}{|ll|}
\hline Engine Specifications & \\
\hline Make & Cummins ISBe220 31 \\
Capacity & $5.9 \mathrm{l}$ \\
Maximum power & $162 \mathrm{~kW}$ at $2000 \mathrm{rpm}$ \\
Maximum torque & $820 \mathrm{Nm}$ at $1500 \mathrm{rpm}$ \\
Number of cylinders & 6 \\
Number of valves per cylinder & 4 \\
Compression ratio & $17.3: 1$ \\
Bore & $102 \mathrm{~mm}$ \\
Stroke length & $120 \mathrm{~mm}$ \\
Dynamometer & Electronically controlled water \\
& brake dynamometer \\
Injection system & Common-rail \\
\hline Data acquisition & \\
\hline Pressure transducer & Kistler piezoelectric transducer \\
& (6053CC60) \\
Analogue-to-digital converter & Data Translation (DT9832) \\
Software & National Instruments LabView \\
Sample rate & 200 kHz \\
Sample time & 4 minutes \\
Data collected & In-cylinder pressure \\
& Band-pass filtered in-cylinder pres- \\
& sure (allowing 4-20 kHz) \\
& Diesel injection timing \\
& Crank-angle rotation information \\
\hline
\end{tabular}


This was achieved with a two channel analogue Krohn-Hite model 3202 filter, by first passing the in-cylinder pressure signal through a high-pass filter with a threshold of $4 \mathrm{kHz}$ and then through a low-pass filter with a threshold of $20 \mathrm{kHz}$. The high-pass threshold was set this close to the frequency of interest, approximately $5-7 \mathrm{kHz}$, to minimise the potential impacts of knocking frequencies. Experiments with the filter settings confirmed that at this threshold the frequency range of interest was unaffected. The low-pass threshold was set to minimise the effects of the cross talk from the diesel injection signal, approximately $28 \mathrm{kHz}$, whilst maintaining as much of the integrity in the signal as possible.

Figure 2 shows an example of the band-pass filtered signal - it is taken from the same cycle as the in-cylinder pressure trace shown in Figure 1. Because this band-pass filtering took place whilst the signal was still in its analogue form, the dependent scale in Figure 2 is in Volts. An advantage of using the technique described is this paper to determine the start of combustion is that it is unnecessary to calibrate the pressure signal. Hence, the processing to convert from the unscaled differential signal to pressure has not been done; from the perspective of the analysis, it would be an extraneous use of computation time.

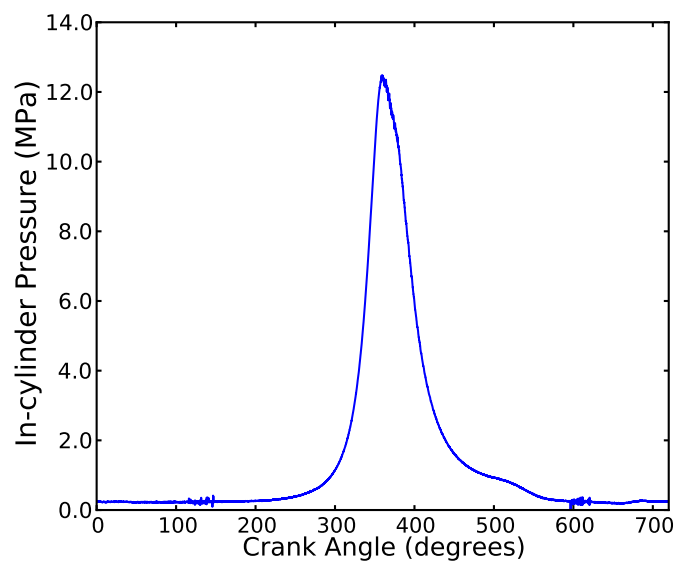

Figure 1: Pressure vs crank angle plot at $2000 \mathrm{rpm}$, full load on neat diesel fuel 


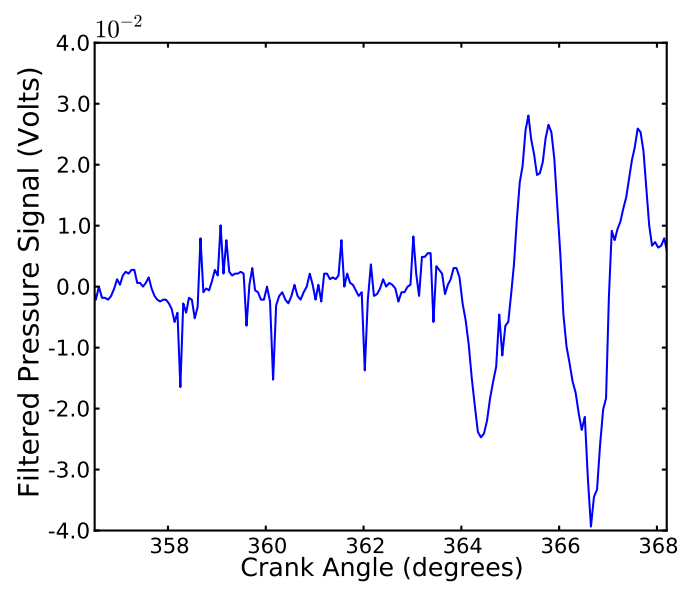

Figure 2: Band-pass filtered pressure signal at $2000 \mathrm{rpm}$, full load on neat diesel fuel

\section{Determination of Ignition Delay}

Ignition delay will be defined as being the time period between the start of diesel injection and the start of combustion [16]. Therefore, in order to determine the ignition delay it is important to obtain accurate knowledge of the diesel injection timing, detailed in Section 4.1, and the start of combustion, detailed from Section 4.3. In the engine setup under investigation in this paper nominal diesel injection timing was able to be determined by directly interrogating the signal sent from the engine management system. For this work, the start of combustion is determined using Bayesian modelling, described in detail from Section 4.3. The Bayesian method described in this paper allows for cycle-by-cycle results to be obtained without any need for cycle averaging.

\subsection{Start of Diesel Injection - Estimating Injection Latency}

Accurate knowledge of the start of fuel injection is important in a study involving ignition delay; however, a common problem is a lack of easily obtainable information regarding injector latency. In the engine setup used in this paper, nominal injection information is obtained by directly interrogating the diesel injection signal sent from the engine management system. Comparative ignition delay studies, such as in this paper, will generally be 
more interested in the differences between test settings rather than absolute knowledge of ignition delay. However, it is still advantageous to consider the injection latency to account for cycle-to-cycle changes in the engine speed.

Figure 3 shows the diesel injection signal super-imposed over net rate of heat release curves of the engine running with neat diesel fuel at full load and of the engine running with cylinder one, where the pressure transducer is located, being motored, both at $2000 \mathrm{rpm}$. The point at which the two net rate of heat release curves deviate from each other will be taken to be the point at which the actual diesel injection begins. Suh and Lee [17] showed in the results of their 2008 study of common-rail diesel injectors that for the injector they investigated latencies as small as approximately $0.25 \mathrm{~ms}$ were normal. Similarly, in a recent study Donkerbroek et al. showed using high speed imaging and by examining a pressure trace of the rail pressure a 3.5 degree crank-angle latency between the injection timing signal and the actual start of injection for their engine setup [7]. In the present engine setup an injection latency of $0.25 \mathrm{~ms}$ correlates exactly with the trough of the dip in the net rate of heat release shown in Figure 3 and the point at which the two net rate of heat release curves deviate. Therefore, it is assumed that the injector latency in this engine setup is $0.25 \mathrm{~ms}$.

Results of the Bayesian analysis shown later in Section 5 will be discussed relative to each other; hence, precisely accurate determination of this value is not completely necessary. However, slight changes in rpm from cycle-tocycle will have a small impact on the number of crank angle degrees which occur in a given time period (i.e. the rate of change of engine speed across 2 consecutive samples may not be precisely the same at each engine cycle during a given test). Therefore, the more accurate the knowledge of the injector latency the better the results will be and hence estimating this latency should not be overlooked. 


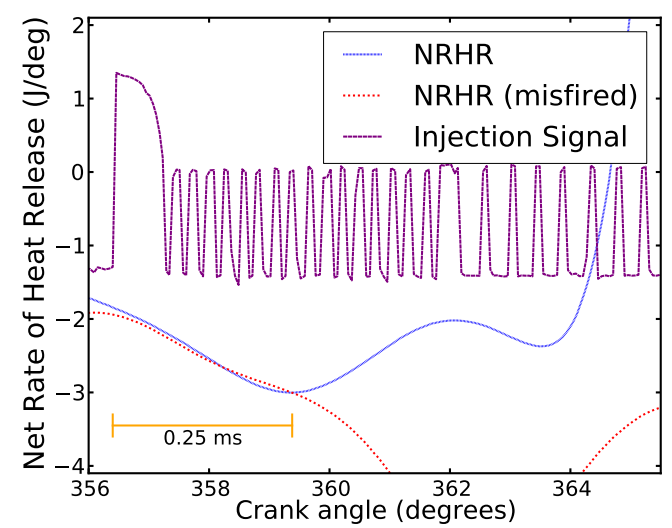

Figure 3: Net rate of heat release: full load with neat diesel and misfired compared to the diesel injection signal

\subsection{Net Rate of Heat Release}

Many authors determine the start of combustion by the analysis of cycleaveraged net rate of heat release curves, such as in Figures 3-5 [18-21]. Inspection of Figure 3 reveals that the start of combustion is at approximately 363.5 degrees crank angle - this result closely correlates to the start of combustion resonance shown in Figure 2. However, even though the traditional net rate of heat release method appears to work well, there are issues with the use of heat release curves for the determination of ignition delay. A few of these issues include $[8,21,22]$ :

- the difficulty in accounting for mixture nonuniformities in the air/fuel ratio and in the burned and unburned gas nonuniformities;

- the effect of crevice regions in the combustion chamber; and,

- assuming the wrong rate of heat transfer, or no heat transfer, between the cylinder charge and combustion chamber walls (especially with the addition of a 'cooling' additive such as water, or a fumigated fuel in a dual-fuel engine).

Moreover, the calculation of the net rate of heat release relies on accurate knowledge of the in-cylinder volume and in-cylinder pressure. In-cylinder volume is analytically determined from crank angle data, although crank 
angle is directly measured there can be inaccuracies in the calibration of top dead centre (TDC). Therefore, the determination of in-cylinder volume is sensitive to the accurate determination of TDC. Figures 4 and 5 show the extent of this sensitivity, Figure 5 is centred around the region of interest to give a clearer indicator of the extent of difference each degree of offset from TDC makes in the calculation of net rate of heat release.

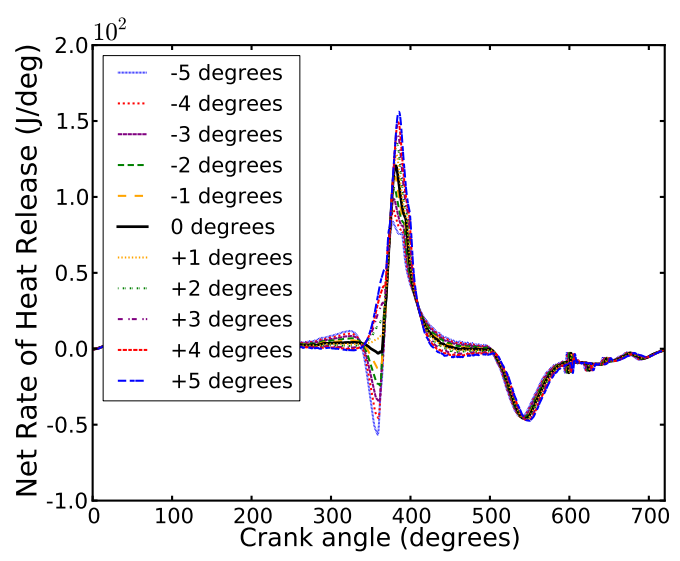

Figure 4: Net rate of heat release curves with the location of TDC displaced \pm 5 crank angle degrees

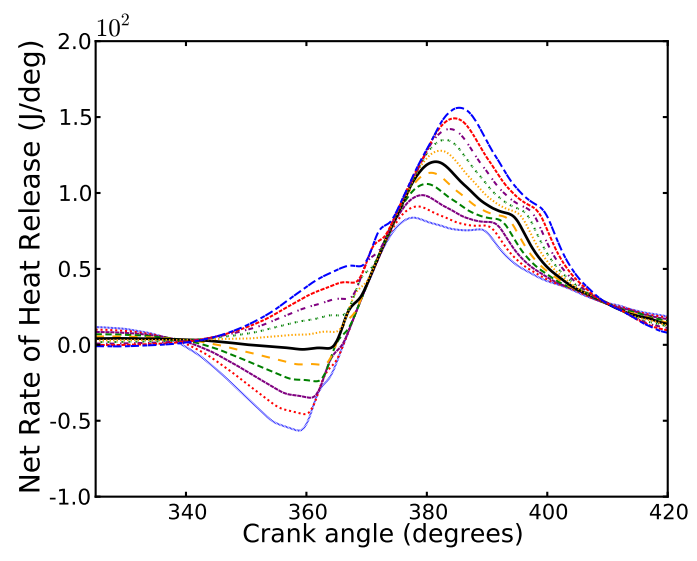

Figure 5: Net rate of heat release curves with the location of TDC displaced \pm 5 crank angle degrees 
Lastly, the need to cycle average, to reduce noise, can potentially skew results if there is a reasonable amount of inter-cycle variability. In the above examples running under standard conditions this is not a large issue because of the engines repeatability from cycle-to-cycle. However, if the engine is run under non-standard conditions, that result in even a moderate amount of inter-cycle variability, this approach has the potential to be problematic. Moreover, the need to cycle average also removes any possibility of exploring the inter-cycle variation that may be present. Therefore, alternative methods should be explored if the engine is run under non-standard conditions, or if the intention of the investigation is to explore inter-cycle variability.

\subsection{Combustion Resonance}

Using combustion resonance to determine the start of combustion overcomes the issues outlined in Section 4.2. As the band-pass filtered in-cylinder pressure is in essence a directly measured quantity, no assumptions or further calculations are required for its generation. This means that combustion resonance can be used for the determination of the start of combustion in any engine condition without concern about the aforementioned issues, particularly those related to post-processing of the in-cylinder pressure data. Work previously done by Bodisco et al. [23] showed that combustion resonance can be modelled using Bayesian statistical modelling. However, their paper focused on the isolation of combustion resonance for the determination of in-cylinder parameters such as temperature and trapped mass. This current work proposes to use a similar approach to determine the start of combustion and, hence, the ignition delay.

For the purposes of this investigation we will qualitatively define the start of combustion as being the point from which the band-pass filtered in-cylinder pressure signal ceases to exhibit only noise-like behaviour and a strong resonance begins $[16,24]$. Therefore, analysis to determine the start of combustion can easily be undertaken manually [16]; however, in order to obtain statistically stable results it would become very time consuming to analyse enough cycles, especially when comparisons between multiple settings are desired. It is proposed that this problem can be overcome with statistical modelling in a Bayesian framework; thus, automating the process of determining the start of combustion. A similar approach has been undertaken by Kim and Min [25] which used wavelet transforms on the block vibration to determine the start of combustion. 
A basic statistical model of the signal in Figure 2 can be used in the determination of the start of combustion. If the assumption that the combustion resonance is built from a stationary frequency - whilst this is not true, over such a small time interval it is true enough for the purposes of this investigation, more complex models require more computation time and will not significantly improve the accuracy of the result $[23,26,27]$ - a very simple model can be used. The conceptual model developed for the determination of the start of combustion is:

$$
\begin{aligned}
y & =s(t) \sim \mathrm{N}(\mu(t), \tau) \\
\mu(t) & =H(t-\delta) A \sin \left(\frac{2 \pi}{\lambda} \omega t+\phi\right),
\end{aligned}
$$

where, $s(t)$, the band-pass in-cylinder pressure signal (such as the example in Figure 2), is assumed to be Normally distributed about some time varying mean, $\mu(t)$, with some standard deviation, $\tau$. The time varying mean, $\mu(t)$, is controlled by a step-function - $\mu$ is zero before the change point defined by

the parameter $\delta$ and when $t>\delta, \mu$ is periodic with a constant amplitude, $A$, sample rate, $\lambda(200 \mathrm{kHz})$, and frequency, $\omega$. Hence using this model, the start of combustion can be determined by resolving the change point parameter, $\delta$.

\subsection{Statistical Model}

A statistical approach can be used to estimate the parameters $\delta, A, \omega$ and $\phi$ from data tuplets $\left\{y_{i}=s\left(t_{i}\right), t_{i} \in \mathcal{T}\right\}$. We estimate these parameters within the Bayesian paradigm to provide marginal posterior plausibility of parameter values based on the data observed e.g. $p(\delta \mid y, t)$. The joint posterior distribution of all parameters is proportional to the product of two terms: the likelihood of the data when parameters are known $p(y \mid \delta, A, \omega, \phi)$; and the priori distribution of all model parameters $p(\delta, A, \omega, \phi)[28]$ :

$$
p(\delta, A, \omega, \phi \mid y) \propto p(y \mid \delta, A, \omega, \phi) p(\delta, A, \omega, \phi)
$$

\subsection{Priors}

A Bayesian approach requires specification of prior plausibility of parameter values, as defined in Section 4.4. If we assume that a priori, what is known about the plausible values of each model parameter, is independent of what is known about all other parameters, then we can factorise 
$p(\delta, A, \omega, \phi)=p(\delta) p(A) p(\omega) p(\phi)$. Moreover, prior distributions should encompass all plausible values a parameter can take [28]. In this investigation vaguely informative priors will be utilised.

Uniform prior distributions can be appropriate in circumstances where there is insufficient knowledge of the nature of the tendency of the model parameter - this is based on Laplace's rationale which has been termed the so-called 'principle of insufficient reason' [28]. Applying the same logic a Uniform prior is assigned to $\phi$. All that is known for a certainty is that $\phi$ could take on any value between 0 and $\pi$, there is no further knowledge about where in this distribution it potentially lies. Therefore, $\phi \sim \operatorname{Unif}(0, \pi)$.

Likewise, examination of the fast Fourier transform of the signal in Figure 2, shown in Figure 6, indicates that the plausible region for the resonant frequency is between $4,500 \mathrm{~Hz}$ and $7000 \mathrm{~Hz}$. Therefore, $\omega \sim \operatorname{Unif}(4500,7000)$. In this case, it could be argued that a Normal distribution would be a more appropriate choice. However, the Uniform distribution was used because of its limiting nature; it was important that the MCMC did not attempt to model a different frequency to the combustion resonance as this would likely result in an undesired posterior distribution for the change point parameter, $\delta$ and hence an incorrect calculation of the start of combustion.

The signal window, such as in Figure 2, was shifted based on an assumed ignition delay so that the start of combustion would be approximately in the middle of the window. Therefore, the start of combustion can be considered unlikely to occur towards the edges of the time period. Here the time period extends for 200 samples ( $\sim 12$ crank angle degrees), so the furthest point from the edges occurs at the centre (at $t=100$ samples). Therefore, in choosing a prior for the change point parameter, $\delta$, a Normal distribution is used to reflect that our uncertainty, centred around the best estimate, behaves like a classical measurement error distribution. By presuming that there is a $95 \%$ chance that the combustion time occurs in the middle half of the time period (i.e. $50 \leq t \leq 150$ ) a prior of $\delta \sim \mathrm{N}(100,25)$ is obtained.

A common aspect of the band-pass filtered signals from this particular engine is that the amplitude is about 0.04 Volts. It is important that the prior for this is reasonably informative; it would not be appropriate if the model parameter, $A$, could be small enough to simply model noise. Much the same, if the amplitude is too large then changes in the other model parameters are unlikely to make any significant difference to the model fit and hence they may not converge, and if they do it may not be to the true value. A Normal distribution has also been selected for $A$ to keep it near to the 0.04 
Volt estimate, but to not be as restrictive as the Uniform distribution for the scenarios where a signal may have a slightly higher or lower amplitude than is expected. The prior distribution does not need to be central around the true value of the parameter as the information about the parameter in the data is stronger [28]. Therefore, the prior is centred about a previous Volt estimate: $A \sim \mathrm{N}(0.04,0.01)$.

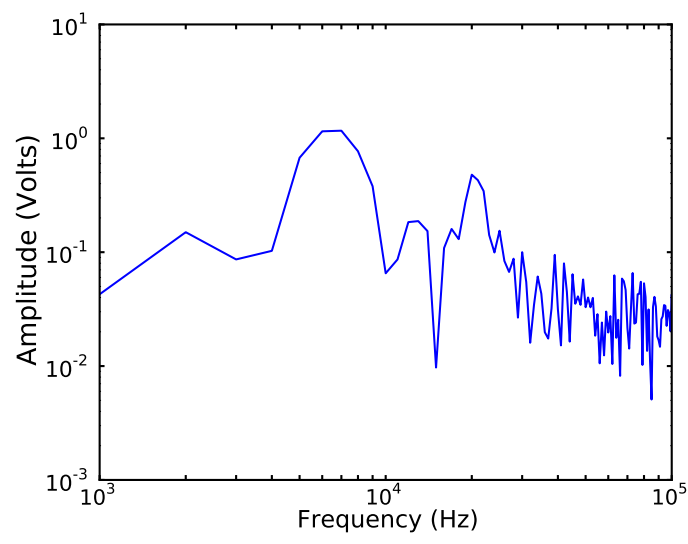

Figure 6: Fast Fourier transform of the band-pass filtered in-cylinder pressure signal in Figure 2

\subsection{Metropolis-Hastings Computational Algorithm}

In order to estimate the posterior distribution of model parameters, we use a standard Bayesian computational approach based on simulation rather than analytic computations. The ergodic theorem ensures that Markov Chain Monte Carlo (MCMC) simulations will in the long run provide a dependent (rather than independent) set of simulations from the posterior distribution [28]. Implementing MCMC through the freely available software package WinBUGS [29] was not found to be computationally fast enough to be feasible. The prepackage software approach was inappropriate mainly due to an emphasis on exploring inter-cycle variability across thousands of consecutive cycles; however, for this type of work it is an acceptable developmental platform.

In situations, such as this, where the posterior cannot be analytically derived, a flexible approach to computation is the Metropolis-Hastings algorithmwhich is a special case of the MCMC algorithm [28]. The Metropolis-Hastings algorithm cycles through two steps. 
1. Propose a candidate value of the parameter from an appropriate proposal distribution $\theta^{*} \sim R(\theta)$.

2. Accept this new proposed value with a probability that depends on its relative plausibility under the posterior distribution, compared to the previous parameter value.

A generic Metropolis-Hastings approach to sampling is selected, where a new value of a parameter is proposed from its full conditional distribution, based on a proposal distribution, and then accepted or rejected using a probability of acceptance that is the minimum of unity and the product of the ratio of proposal likelihoods and the ratio of posterior probabilities evaluated at the old and new proposed value.

$$
\alpha=\min \left\{1, \frac{R\left(\theta^{*}\right)}{R\left(\theta^{m-1}\right)} \frac{p\left(\theta^{*} \mid \cdot\right)}{p\left(\theta^{m-1} \mid \cdot\right)}\right\}
$$

where, $m=1, \ldots, M$ denotes the $m^{\text {th }}$ MCMC simulation from the chain, $\frac{R\left(\theta^{*}\right)}{R\left(\theta^{m-1}\right)}$, the proposal ratio, is selected to evaluate to unity and,

$$
p(\theta \mid \cdot)=p(\theta) p(y \mid \theta ; \cdot)
$$

where, - denotes the full set of parameters $\left\{\mu_{t} ; \delta, A, \omega, \phi\right\}$ omitting the parameter of interest, here generically denoted as $\theta$. Therefore,

$$
\theta^{m}= \begin{cases}\theta^{*} & \text { with probability } \alpha \\ \theta^{m-1} & \text { otherwise }\end{cases}
$$

Each candidate parameter, denoted as $\theta^{*}$, is selected by a cyclic sampling strategy from its conditional posterior distribution with the existing value of the parameter, $\theta^{m-1}$, as the mean [28].

$$
\theta^{*} \sim \mathrm{N}\left(\theta^{m-1}, \sigma_{\theta}\right)
$$

Hence $\sigma_{\theta}$ acts as a tuning parameter, which determines how far the algorithm seeks for candidate parameter values at each step. A large value of $\sigma_{\theta}$ promotes large steps but could provide many wasted proposals. Conversely, a value of $\sigma_{\theta}$ that is too small promotes small steps that may take longer to converge towards the true value, and may also induce high cross-correlation 
of parameter values. Selection of the candidate parameter for $\delta$ is done similarly; however, instead of sampling from a Normal distribution a Uniform distribution is used:

$$
\delta^{*} \sim \operatorname{Unif}\left(\delta^{m-1}-n, \delta^{m-1}+n\right)
$$

where, $n$ defines the maximum possible deviation the candidate parameter for $\delta$ can have from the existing value of $\delta$ - the candidate parameter is selected from the integers in $\mathrm{Z}$ that fall within $n$ units of $\delta^{m-1}$. The use of a Uniform proposal distribution for $\delta$ is to ensure broad exploration.

\subsection{Simulation Results}

After a suitable burn-in time (in this case 2000 iterations) model parameters are saved in an array (in this case 200 values, every fifth value out of 1000 iterations) to create a posterior density for each model parameter. Examination of the kernel density estimate (probability density function) for each parameter, the posterior density, will not only yield the most plausible value (taken to be the posterior mode), but also give the analyst an indication of the uncertainty in the result $[28,30,31]$. From the analysis of the signal shown in Figure 2, it can be seen in Figure 7 that the most plausible start of combustion timing is $363.97^{\circ}$ with a $95 \%$ credible interval of [363.78, 364.14]. Similarly, the analysis also shows that the most plausible initial resonant frequency (Figure 8) is $6215 \mathrm{~Hz}$ with a $95 \%$ credible interval of $[6202,6233]$.

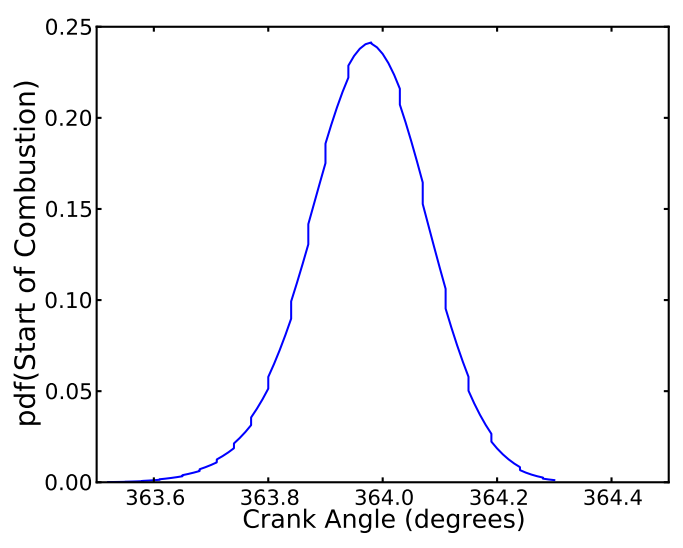

Figure 7: Posterior density of the start of combustion, $\delta$, of the band-pass filtered in-cylinder pressure signal in Figure 2 


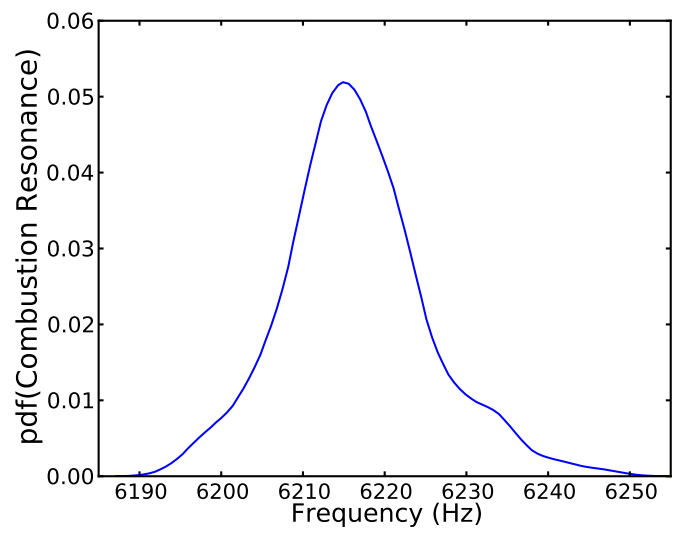

Figure 8: Posterior density of the combustion resonance, $\omega$, of the band-pass filtered in-cylinder pressure signal in Figure 2

Reconstructing the signal from the model allows the analyst to visually determine if the model outputs are feasible. Figure 9 shows the signal in Figure 2 with a visualisation of the model, as described in Equation 3. Parameter estimates for this cycle are shown in Table 2 . It can be visually seen that the model and the signal agree well, especially in relation to the change point parameter. This is evident by how closely the model follows the signal it was fitted to: the resonant frequency, $\omega$, matches that of the signal and the periodic part of the model begins at the same time as the combustion resonance.

Another outcome of this simulation is an estimate of the initial resonant frequency, $\omega$-as it is only fitting across a small section of the data the assumption of a stationary frequency will not be significantly incorrect enough to produce a misleading result. In the case of Figure 3, two periods of the combustion resonance are present, if it were much more than this the assumption of a stationary frequency would fail and a misleading result may be obtained. Therefore, the model described in this paper can be used effectively as a means of determining the start of combustion and the initial resonant frequency. 
Table 2: Model priors and parameter estimates of the band-pass filtered in-cylinder pressure signal shown in Figures 2 and 9 based on the model described by Equation 3

\begin{tabular}{lllll} 
Variable & $\begin{array}{l}\text { Prior } \\
\text { Mean }\end{array}$ & $\begin{array}{l}\text { Prior } \\
\text { StDev }\end{array}$ & $\begin{array}{l}\text { Posterior } \\
\text { Mode }\end{array}$ & $\begin{array}{l}\text { Posterior } \\
\text { StDev }\end{array}$ \\
\hline$A$ (Volts) & 0.04 & 0.01 & 0.024 & 0.001 \\
$\delta\left(^{\circ}\right.$ crank angle $)$ & 100 & 20 & 127 & 1 \\
& $\left(362.34^{\circ}\right)$ & $\left(\approx 1.52^{\circ}\right)$ & $\left(363.97^{\circ}\right)$ & $\left(0.05^{\circ}\right)$ \\
\hline Uniform Prior & Low & High & & \\
$\omega($ Hz $)$ & 4500 & 7000 & 6215 & 9 \\
$\phi$ & 0 & $\pi$ & 2.92 & 0.03 \\
\hline
\end{tabular}

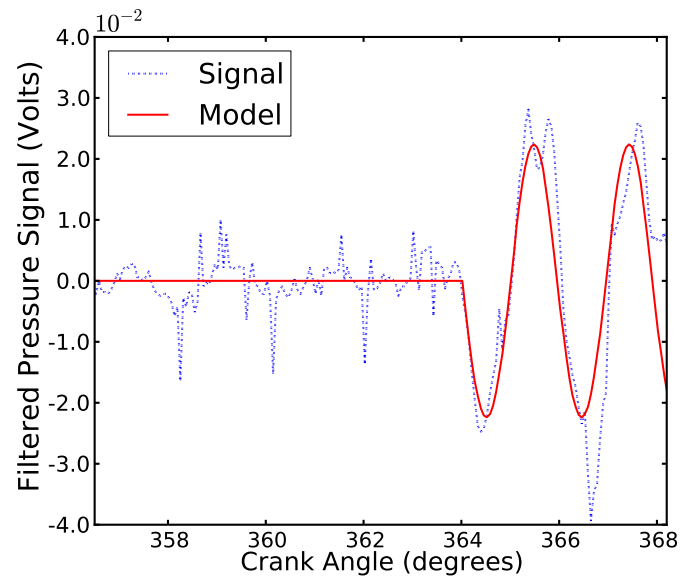

Figure 9: Model of the band-pass filtered in-cylinder pressure signal in Figure 2

\section{Inter-cycle Variability}

An important justification for using the analysis methodology described in the previous section was to allow for cycle-by-cycle analysis of the data. As the Bayesian method is reliable with noisy data the need to cycle average is removed and individual cycles can be examined independently. Hence, the data can be investigated across large numbers of consecutive cycles - the results shown in the section are from 4000 consecutive cycles (4 minutes of 
data). This investigation was performed on a standard desktop computer in $\mathrm{C}++$ and the analysis took approximately 15 seconds per cycle to perform.

Ignition delay is influenced by the injection timing because of changes in the charge air temperature and pressure [8]. The empirical relationship for estimating ignition delay given by Hardenberg and Hase [11] in Equation 2 shows the temperature and pressure dependence at the time of injection. Therefore, no ignition delay study would be complete without first examining the injection timing. Figure 10 shows the injection timing - this data is presented in a histogram because of the discrete nature of the diesel injection timing. The injection timing is controlled by the engine management system, in all test cases the injection strategy was one a single sustained injection and combustion occurred before the diesel injection was completed.

Advanced injection timing, such as exhibited by the engine under investigation, is common among modern diesel engines to mitigate harmful emission $[32,33]$. Improved Emission control is achieved with advanced injection timing because of an increase in premixed combustion and subsequent changes to in-cylinder pressure and decreased in-cylinder temperature [34]. Nitrogen oxides (NOx), along with particulate matter, are a key emission from diesel engines. Excessive NOx formation is normally related to high in-cylinder temperature and excess oxygen [35] and is therefore directly influenced by the injection timing, injection pressure and the amount of mixing prior to combustion, and hence the ignition delay.

Knowledge of the injection timing is determined from interrogating the diesel injector signal and is corrected for injector latency, as explained in Section 4.1. At half load the diesel injection timing is significantly later than at full and three quarter load. There is more than a crank-angle degree difference between the start of diesel injection for half load when compared with three quarter and full load. At half load injection typically occurs at approximately 360.9 degrees crank-angle, whereas at three quarter load injection typically occurs at approximately 359.7 crank-angle degrees and at full load injection typically occurs at approximately 359.3 degrees crankangle.

Figure 11 shows the combustion timing, $\delta$. Similar to the injection timing shown in Figure 10, full and three quarter load commence combustion at a similar time, approximately 365 degrees crank-angle for three quarter load and approximately 364 degrees crank-angle for full load, whereas the start of combustion for half load is much later at approximately 368 degrees crankangle. Also worthy of noting is that, as the load is decreased the inter- 
cycle variability of the diesel injection timing and the start of combustion is increased. This is most likely an artefact of the stability of the in-cylinder temperature. At half load the inter-cycle variability is considerably increased when compared to the higher loads, the inter-cycle variability at full and three quarter load is very similar with only a fractional increase as the load was decreased. This is also clearly seen in the ignition delay results, shown in Figure 12. The ignition delay at half load is approximately two degrees crank-angle longer than at full and three quarter loads, and significantly less stable. Both Figures 11 and 12 show that on some cycles at half load combustion occurs much later than the rest of the cycles. This is likely caused by fluctuations in the in-cylinder temperature. Including these cycles which are exhibiting unusual behaviour does not significantly change the calculation of ignition delay - the modal, mean and median ignition delay at half load is 6.9 crank-angle degrees, at three quarter load the ignition delay is 5.2 crank-angle degrees and at full load the ignition delay is 4.8 crank-angle degrees.

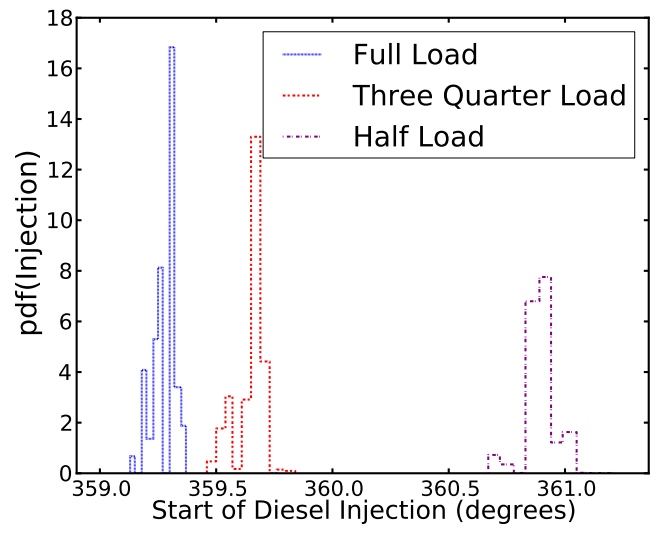

Figure 10: Diesel injection timing 


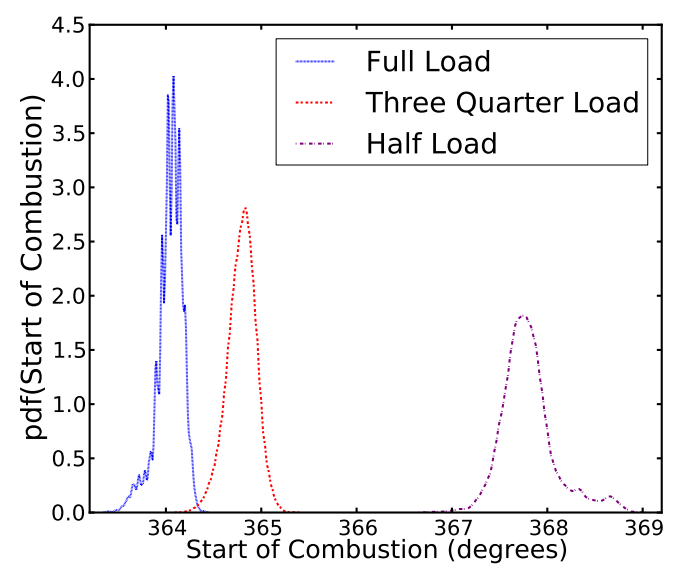

Figure 11: Start of combustion as determined from the combustion resonance, parameter $\delta$

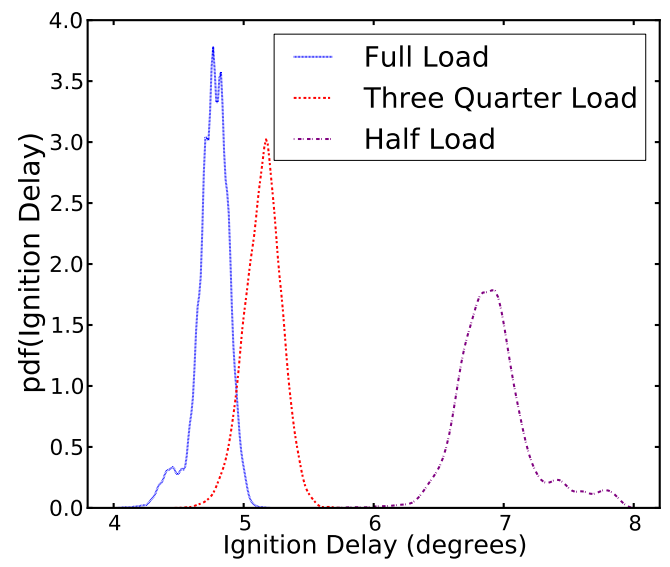

Figure 12: Ignition delay as determined from the combustion resonance

\section{Comparison to Net Rate of Heat Release}

Traditionally the start of combustion was determined by examining a net rate of heat release plot [8] - the start of combustion is usually characterised to be the point at which the net rate of heat release becomes positive. Figure 13 shows a net rate of heat release plot generated from the average of 4000 
engine cycles at $2000 \mathrm{rpm}$ and full load using Equation 1. On the opposing axis of Figure 13 is the start of combustion distribution shown in Figure 11, generated from the same 4000 engine cycles as the net rate of heat release. Good agreement between the two methods can be seen; the start of combustion, as determined by the Bayesian analysis, lines up well with the start of the increase in net rate of heat release at approximately 364 degrees crankangle. Also of note in Figure 13 is that the start of combustion distribution, from the Bayesian analysis described in this paper, lines up with the start of the increase in the net rate of heat release, rather than when the net rate of heat release becomes positive.

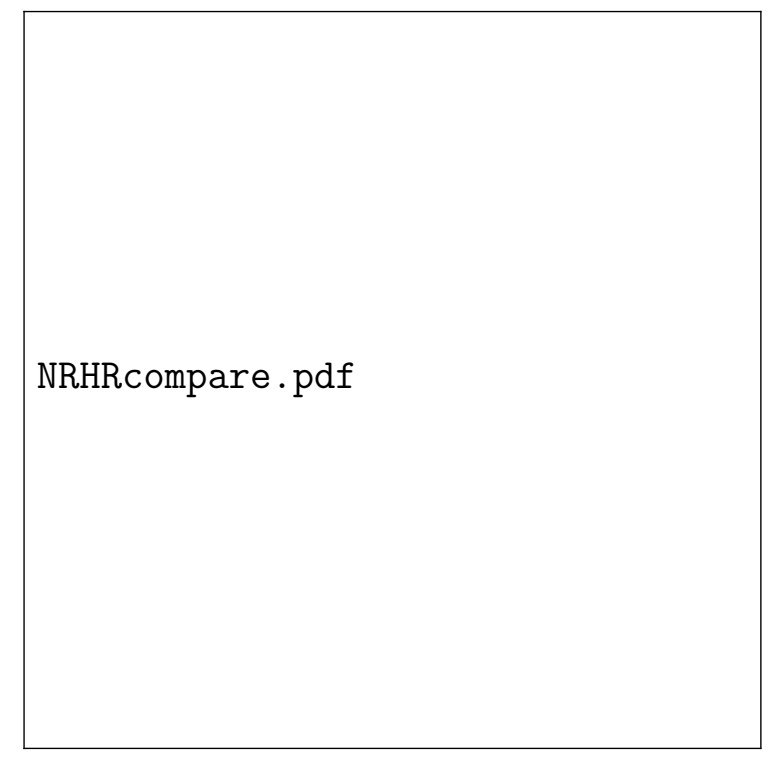

Figure 13: Net rate of heat release and start of combustion as determined from the combustion resonance, parameter $\delta$, at full load

\section{Conclusion}

This paper has introduced a powerful new method for determining ignition delay. In contrast to standard techniques this method uses combustion resonance, in the form of band-pass filtered in-cylinder pressure, and Bayesian statistical modelling to accurately resolve the start of combustion. It was also demonstrated that this method allows for true inter-cycle variability studies and does not suffer from some of the issues surrounding the use of in-cylinder 
pressure directly to determine the start of combustion. The results shown in this paper demonstrate the utility of this technique by examining 4000 consecutive cycles at 3 different engine loads: full, three quarter and half loads. At half load the ignition delay was much longer than at the higher loads and the inter-cycle variability was greatly increased when compared to the higher loads.

The Bayesian modelling method shown in this paper for determining the start of combustion uses in-cylinder pressure. In-cylinder pressure methods are optimal because they are inexpensive, when compared to optical sensors; also, using a measured engine parameter that is directly related to the engine work for a study into ignition delay makes intuitive sense. Moreover, this is the only in-cylinder pressure technique currently available for determining ignition delay that does not involve differentiation. Differentiating noisy data decreases the signal-to-noise ratio and therefore complicates sensitive analysis such as this. Further, Bayesian modelling requires only a small number of data points to be effective and is not adversely effected by noise. Therefore, the methodology shown in this paper for determining the start of combustion can be used to accurately resolve the ignition delay for individual cycles, which is important if inter-cycle variability studies are required.

\section{Acknowledgments}

The authors wish to thank Mr. Anthony Morris for assisting with the design of the experimental campaigns and his invaluable technical knowledge and Mr. Noel Hartnett for his work in maintaining the engine for research. Further thanks also to Technologist Mr. Ken McIvor for his assistance in setting up the data acquisition software. This work was undertaken under an Australian Research Council Linkage Grant (LP0775178) in association with Peak3 P/L.

\section{References}

[1] R. Papagiannakis, D. Hountalas, Experimental investigation concerning the effect of natural gas percentage on performance and emissions of a DI dual fuel diesel engine, Applied Thermal Engineering 23 (3) (2003) 353-365.

[2] Y. Ren, Z. Huang, D. Jiang, L. Liu, K. Zeng, B. Liu, X. Wang, Combustion characteristics of a compression-ignition engine fuelled with diesel- 
dimethoxy methane blends under various fuel injection advance angles, Applied Thermal Engineering 26 (4) (2006) 327-337.

[3] J. Ghojel, D. Honnery, K. Al-Khaleefi, Performance, emissions and heat release characteristics of direct injection diesel engine operating on diesel oil emulsion, Applied Thermal Engineering 26 (17-18) (2006) 2132-2141.

[4] P. Sahoo, L. Das, Combustion analysis of Jatropha, Karanja and Polanga based biodiesel as fuel in a diesel engine, Fuel 88 (6) (2009) 994-999.

[5] C. Cinar, O. Can, F. Sahin, H. S. Yucesu, Effects of premixed diethyl ether (DEE) on combustion and exhaust emissions in a HCCI-DI diesel engine, Applied Thermal Engineering 30 (4) (2010) 360-365.

[6] D. Lata, A. Misra, Analysis of ignition delay period of a dual fuel diesel engine with hydrogen and LPG as secondary fuels, International Journal of Hydrogen Energy 36 (5) (2011) 3746-3756.

[7] A. Donkerbroek, M. Boot, C. Luijten, N. Dam, J. ter Meulen, Flame lift-off length and soot production of oxygenated fuels in relation with ignition delay in a DI heavy-duty diesel engine, Combustion and Flame 158 (3) (2011) 525-538.

[8] J. B. Heywood, Internal Combustion Engine Fundamentals, McGrawHill, Inc., 1988.

[9] R. Stone, Introduction to Internal Combustion Engines, Macmillan Press Ltd, London, 1999.

[10] D. Rothamer, L. Murphy, Systematic study of ignition delay for jet fuels and diesel fuel in a heavy-duty diesel engine, Proceedings of the Combustion Institute (2012).

[11] H. O. Hardenberg, F. W. Hase, An empirical formula for computing the pressure rise delay of a fuel from its cetane number and from the relevant parameters of direct-injection diesel engines, SAE Technical Paper 790493 (1979).

[12] G. Prakash, A. B. Shaik, A. Ramesh, An approach for estimation of ignition delay in a dual fuel engine, SAE Technical Paper 1999-01-0232 (1999). 
[13] D. N. Assanis, Z. S. Filipi, S. B. Fiveland, M. Syrimis, A predictive ignition delay correlation under steady-state and transient operation of a direct injection diesel engine, J. Eng. Gas Turbines Power 125 (2) (2003) 450-457.

[14] R. Payri, J. M. Garcia-Oliver, M. Bardi, J. Manin, Fuel temperature influence on diesel sprays in inert and reacting conditions, Applied Thermal Engineering 35 (0) (2012) 185-195.

[15] A. Ellison, Bayesian inference in ecology, Ecology Letters 7 (6) (2004) 509-520.

[16] T. Bodisco, R. J. Brown, Inter-cycle variability of in-cylinder pressure parameters in an ethanol fumigated common rail diesel engine, Energy 1 (52) (2013) 55-65.

[17] H. K. Suh, C. S. Lee, Experimental and analytical study on the spray characteristics of dimethyl ether (DME) and diesel fuels within a common-rail injection system in a diesel engine, Fuel 87 (6) (2008) 925-932.

[18] D. A. Kouremenos, C. D. Rakopoulos, K. G. Kotsos, A stochasticexperimental investigation of the cyclic pressure variation in a DI singlecylinder diesel engine, International Journal of Energy Research 16 (9) (1992) 865-877.

[19] C. Rakopoulos, K. Antonopoulos, D. Rakopoulos, Experimental heat release analysis and emissions of a HSDI diesel engine fueled with ethanoldiesel fuel blends, Energy 32 (10) (2007) 1791-1808.

[20] M. Shehata, Cylinder pressure, performance parameters, heat release, specific heats ratio and duration of combustion for spark ignition engine, Energy 35 (12) (2010) 4710-4725.

[21] X. Tauzia, A. Maiboom, S. R. Shah, Experimental study of inlet manifold water injection on combustion and emissions of an automotive direct injection diesel engine, Energy 35 (9) (2010) 3628-3639.

[22] M. Brunt, K. Platts, Calculation of heat release in direct injection diesel engines, SAE Technical Paper 1999-01-0187 (1999). 
[23] T. Bodisco, R. Reeves, R. Situ, R. J. Brown, Bayesian models for the determination of resonant frequencies in a DI diesel engine, Mechanical Systems and Signal Processing 26 (2012) 305-314.

[24] A. P. Carlucci, F. F. Chiara, D. Laforgia, Block vibration as a way of monitoring the combustion evolustion in a direct injection diesel engine, SAE Paper 2006-01-1532 (2006).

[25] S. Kim, K. Min, Detection of combustion start in the controlled auto ignition engine by wavelet transform of the engine block vibration signal, Measurement Science and Technology, 19 (8) (2008).

[26] R. Hickling, D. A. Feldmaier, F. H. K. Chen, J. S. Morel, Cavity resonances in engine combustion chambers and some applications, Acoustical Society of America Journal 73 (1983) 1170-1178.

[27] Y. Ren, R. B. Randall, B. E. Milton, Influence of the resonant frequency on the control of knock in diesel engines, Proceedings of the Institution of Mechanical Engineers: Part D : Journal of Automobile Engineering. London 213 (2) (1999) 127-133.

[28] A. Gelman, J. B. Carlin, H. S. Stern, D. B. Rubin, Bayesian Data Analysis: Second Edition, Chapman \& Hall/CRC, 2003.

[29] D. Spiegelhalter, A. Thomas, N. Best, WinBUGS Version 1.2 User Manual, MRC Biostatistics Unit (1999).

[30] M. Rosenblatt, Remarks on some nonparametric estimates of a density function, The Annals of Mathematical Statistics 27 (3) (1956) 832-837.

[31] E. Parzen, On estimation of a probability density function and mode, The Annals of Mathematical Statistics 33 (3) (1962) 1065-1076.

[32] D. Choi, P. C. Miles, H. Yun, R. D. Reitz, A parametric study of lowtemperature late-injection combustion in a HSDI diesel engine, JSME International Journal Series B 48 (4) (2005) 656-664.

[33] C. L. Genzale, R. D. Reitz, M. P. B. Musculus, Effects of spray targeting on mixture development and emissions formation in late-injection lowtemperature heavy-duty diesel combustion, Proceedings of the Combustion Institute 32 (2) (2009) 2767-2774. 
[34] B. Prabhakar, A. L. Boehman, Effect of common rail pressure on the relationship between efficiency and particulate matter emissions at NOx parity, SAE Paper 2012-01-0430 (2012).

[35] G. A. Lavoie, J. B. Heywood, J. C. Keck, Experimental and theorectical study of nitric oxide formation in internal combustion engines, Combustion Science and Technology 1 (1970) 313-326. 